like symptoms,movement disorders and altered state of consciousness.Neuron spesific antibodies (LGI1, CASPR2, AMPAR1/2, NMDAR, GABABR) in CSF/serum, antibodies against GAD, thyroid peroxidase and paraneoplastic syndrome panel(anti-Hu, anti-Yo, anti-Ri, anti-Amphipysian, anti-Tr, antiPCA-2, anti-Ma, anti-CV-2, anti-ANNA-3) were detected in the serum.

Results The mean onset age of patients were 8,3 years (213,8 years). Female and male ratio was $8: 7$. In all patients admitted to the hospital the most common symptom was seizure $(73 \%)$. The other symptoms were psychiatric(20\%) and altered state of consciousness(7\%). Seven(46\%) patients with acute seizures present with status epilepticus. The mean hospitalization time of patients were 38,5 days (14-70 days) and 20 days(3-50) at Intensive Care Unit. Five patients were mechanically ventilated.

Significant serum levels of antibodies were detected in 10 out of 15 patients.(GAD $\mathrm{n}=8$, antiTPO $\mathrm{n}=5$ ) CASPR2 and NMDA in CSF were detected in different two patients. One patient diagnosed with FIRES.Oligoclonal banding or elevetad IgG index were detectected in 6 of 15 patients. Lymphocytic pleocytosis $(40 \%)$ were detected in cerebrospinal fluid.

All patients had abnormal electroencephalogram findings but $35.7 \%$ of patients had abnormal brain magneticresonance imaging findings at the time admission of the hospital.

Immunotherapy,firstline therapy (IVIG $n=14$, pulse steroid $n=13$, plasma exchange $n=7$ ) and second line therapy (rituximab $n=1$, cyclophosphamide $n=1$ ) were contributed to treatment .

After the discharge of the hospital no more seizure were seen in 12 patients $(80 \%)$ during follow-up period. At the last visit all of these patiens had normal EEG findings. But in the other 3 patients epileptic activity was detected in their EEGs. Among them,only one patient was resistant to the antiepileptic treatment.

Conclusions In this study, we observed that seizures with IME were generaly resistant to the treatment during the acute phase of the illness in pediatric population. But after proper and early immunotherapies, seizures remission was detected with good prognosis in most of them.

\section{P493 LATE AND ATYPICAL PRESENTATION OF MECP2 MUTATION}

${ }^{1}$ Karen Kelleher*, ${ }^{2}$ Sally Ann Lynch, 'Bryan Lynch. 'Department of Neurology, Temple Street Childrens University Hospital, Dublin, Ireland; '2Department of Clinical Genetics, Temple Street Childrens University Hospital, Dublin, Ireland

10.1136/archdischild-2019-epa.829

Introduction Rett syndrome (RS) is an X-linked dominant neurodevelopmental disorder affecting girls, most commonly caused by MECP2 loss-of-function mutations. The typical phenotype of RS is characterised by a period of developmental stagnation in late infancy, followed by progressive dementia, developmental regression, stereotyped hand-movements, microcephaly and gait dyspraxia. There are well recognised variants such as Atypical Rett syndrome(Angelman-like), Zappella-variant(preserved speech variant), and Congenital Rett syndrome. Objective We describe two cases of unrelated Irish females who presented towards the end of their first decade with unusual Rett phenotypes.

Results Both cases had normal early development. Mild/moderate developmental abnormalities were picked up at 2 years.
Prior to onset of epilepsy, both cases were in mainstream school with a special needs assistant and had upright and independent gait. Case 1 developed severe progressive scoliosis from preschool age.

Case 1 and Case 2 presented with severe, refractory epilepsy aged 10 and 9 respectively; consisting of a mixture of atonic and complex-partial seizures evolving to include epileptic-spasms with epileptic-encephalopathy in both. In both cases gait deterioration began as an increasingly crouched posture and shuffling gait progressing to wheelchair-dependence. Concurrently, was a profound regression in motor skills, cognitive abilities, very poor appetite and weight loss. Neither had features of hand-wringing or microcephaly.

Case 1 was notable for excessive drowsiness and development of progressive neuromuscular muscle weakness leading to frequent pneumonias, life-threatening choking episodes and a progressive deterioration in her respiratory function. She died, aged 13 years, of respiratory failure. Case 2, aged 13, does not have features of progressive bulbar palsy described in Case 1.

Extensive workup was unrevealing except for de-novo heterozygous mutations in their MECP2 genes. In Case 1, a novel variant of MECP2 was detected: A duplication of the majority of the protein-coding portion of exon 4. Isolated duplication of large sections exon 4 has never, to our knowledge, been described before. In Case 2 there was a deletion and seven base pair insertion in exon 4:1006 1275delinsBATGCCC(p.Leu336Aspfs*12), predicted to introduce a premature termination codon.

Conclusions Case 1 represents a unique case of MECP2 mutation, expanding our knowledge of the deleterious mutations leading to a Rett phenotype.

We suggest that young girls with late onset developmental regression, new onset refractory epilepsy and crouching gait be investigated for MECP2 gene mutations.

Early onset of scoliosis in an ambulatory child, and severe neuromuscular weakness are not previously known features of this condition.

\section{P494 ETIOLOGY OF MICROCEPHALY: 5 YEARS EXPERIENCE}

Şule Akyan*, Eda UTiNE. Hacettepe, Ankara, Turkey

\subsection{6/archdischild-2019-epa.830}

Microcephaly is a developmental disorder in which the head circumference, measured as an occipitofrontal, is below the third circumferential, or at least 2 standard deviations (SD), relative to age, gender, and ethnic origin. The aim of this study is to evaluate the diagnostic approach of microcephaly in childhood and to determine the prevalence of various underlying causes/diseases, and to determine a standard diagnostic approach according to disease frequency. 1474 patients who had microcephaly and from prenatal 32 nd gestational week to 18 years of age was admitted to any of the outpatient clinics of Hacettepe University Hospitals between July 2012 and July 2017. The patients' head circumference distribution (SDS) was $-2 /-3$ with a rate of $24.97 \%$, the ratio of patients with $-3 /-4$ was $30.6 \%$, the ratio of patients with $-4 /-5$ was $18.3 \%$ and $-5 /-$ The rate of patients with 6 was $11.87 \%$. According to the majority of patients, $68,72 \%$ were genetic, $4,21 \%$ were related with chronic disease, $3,73 \%$ were related to metabolic disease 
and $3,39 \%$ were found to have a perinatal/antenatal brain injury etiology. Of the 906 patients who had records for the seizure history, 39\% were diagnosed with epilepsy and $93.77 \%$ of these patients had clinical seizures and an electroencephalography-determined seizure. The rate of global growth retardation was determined as $88.6 \%$ in the patients with developmental evaluation abnormal by DENVER II developmental screening test. When DENVER II findings of the patients with growth retardation were evaluated in terms of area, it was found that $91.1 \%$ of the patients had language, $82.7 \%$ had personal and social, $89.3 \%$ had gross motor and $87.1 \%$ had thin motor field regression. The patients with WISC-R were found to have a mild intellectual disability rate of $51.1 \%(\mathrm{n}=24)$, a moderate mental disability rate of $44.7 \%(n=21)$ and a severe mental deficiency rate of $4.3 \%(\mathrm{n}=2)$ determined. $29.72 \%$ of patients who underwent cranial MRI showed abnormal findings. Cerebral anomaly was found in $94.3 \%$ of patients with abnormal cranial MRI findings, $16.7 \%$ had brain stem dysplasia, $11.6 \%$ had cerebellar anomalies, $1.4 \%$ had pituitary gland hypoplasia and $0.2 \%$ had meningoencephalocele were detected. Therefore, microcephalic patients should be closely monitored in terms of epilepsy, mental insufficiency and global growth retardation, and counseling should be provided to their families. In addition, it was found that cranial MRI was important for the clarification of some etiologies.

\section{P495 IS NARCOLEPSY INCIDENCE INCREASING OR SYMPTOMS JUST BETTER RECOGNISED?}

${ }^{1}$ Jennifer Hayden*, ${ }^{2}$ Lisa Dann, ${ }^{2}$ Nicolas M Allen, ${ }^{3}$ Bryan Lynch. ${ }^{1}$ Dept. of Paediatrics, National University of Ireland Galway, and Galway University Hospita, Galway, Ireland; ${ }^{2}$ Galway University HospitalDept. of Paediatrics, National University of Ireland Galway, and Galway University Hospital (GUH), Galway, Ireland; ${ }^{3}$ Galway University HospitalTemple St. Children's University HospitalTemple St. Children's University Hospital Temple St. Children's University Hospital, Dublin , Ireland

10.1136/archdischild-2019-epa.831

Background Narcolepsy is a rare and under-recognised disease characterised by excessive daytime sleepiness, disturbed nocturnal sleep, hallucinations, and cataplexy, with a major impact on quality of life. Diagnosis can be challenging. We present a series of recent children presenting to a district general hospital over a 2.5 year period with chronic symptoms ultimately diagnosed with narcolepsy.

Patients Patient 1: Female(12y) GP referral with 2-year history of increased sleepiness and falling asleep during meals. Later developed cataplexy-'when I laugh, I go weak suddenly'. Swine-flu vaccine 6 years previously.

Patient 2: Female(8y) GP referral with 18-month history of excessive day-time sleepiness and vivid nightmares. Narcolepsy was considered when seen in clinic initially but significant academic decline subsequently prompted formal diagnosis (9 months after referral). Laugh induced catatonia (eyes roll, tongue protrusion and lower limb weakness). Pandemrix H1N1 vaccine 3 years previously. Sleep study noted abnormal sleep latency and sleep onset REM periods. Improvement with methylphenidate.

Patient 3: Male(10y) emergency department (ED) presentation with a 2-month history of excessive day time sleepiness and increased appetite. Falling asleep while standing occasionally. No cataplexy. Sleep study abnormal (2.3 minutes sleep latency and SOREM periods in $3 / 4$ naps). Treatment with sodium oxybate.

Patient 4: Female(5y) ED presentation with 2-month history of daily episodes of eye rolling and sudden collapse, weeks following MMR. Initial diagnosis and treatment for epilepsy. Video review reveals clinical diagnosis of cataplexy; focused history revealed excessive daytime sleepiness. Treatment with stimulants (methylphenidate) improved sleep symptoms; considering sodium-oxybate for cataplexy.

Patient 5: Male(15y) GP referral with 3 year history of excessive day-time sleepiness, struggling to keep up academically. Upon laughing his mouth droops open with tongue protrusion (cataplexy). No swine-flu vaccine. Treatment pending investigations.

Patients 1-4 had undetectable CSF hypocretin levels (except patient 2; failed LP) and positivity for the HLA-DQB1*0602 association in keeping with narcolepsy. Patient 5 is awaiting specific investigations. Sleep studies are pending for 3 patients. Secondary causes of narcolepsy were out-ruled.

Discussion Delay in narcolepsy diagnosis is associated with worse outcomes. While narcolepsy is thought to be under recognised, these 5 patients were suspected upon referral to paediatrics and management instigated quickly; however focused history and increased awareness is required. Our series and review of a national narcolepsy clinic suggests increased incidence and early detection in children. A national narcolepsy service (currently Temple-St-Hospital, Dublin) facilitates management, as symptoms and co-morbidities can be debilitating and life-long. Vaccine association remains controversial.

\section{P496 X- LINKED INFANTILE SPINAL MUSCULAR ATROPHY (SMAX2) CAUSED BY NOVEL C.1681G>A SUBSTITUTION IN THE UBA1 GENE, EXPANDING THE PHENOTYPE}

Niamh Shaughnessy*, Eva B Forman, Declan O’ Rourke, Sally Ann Lynch, Lynch Bryan. Temple Street Children's University Hospital , Dublin, Ireland

10.1136/archdischild-2019-epa.832

Aims We report the case of a male infant who presented following a normal pregnancy with typical symptoms of X-linked infantile spinal muscular atrophy including hypotonia, weakness, areflexia and respiratory insufficiency, however contractures were absent. X-linked infantile spinal muscular atrophy (SMAX2), OMIM 301830, is a rare, severe form of spinal muscular atrophy, caused by variants in the Ubiquitin like modifier-activating enzyme 1 (UBA1) gene1. Clinical features reported to date include marked hypotonia, areflexia, arthrogryposis, contractures, myopathic facies, tongue fibrillations and cryptorchidism2. To our knowledge, this is the first reported case of SMAX2 to present without contractures.

Methods This infant was born at $40+4$ weeks following a normal pregnancy to non-consanguineous Irish parents. Intermittent stridor was noted following delivery, laryngomalacia was diagnosed following review by ENT. At 8 weeks old there was an acute event at home with colour change and poor feeding for 2 days. On presentation to ED, there was marked increased work of breathing, weakness, absent deep tendon reflexes, hypotonia, myopathic facies and weak cry. Eyes were bright and there was no ophthalmoplegia or contractures. A significant family history of neuromuscular disease on the patient's maternal side was noted, with several male relatives all dying before the age of six months. Creatine Kinase was 